\title{
La qualité bactériologique des eaux de consommation (forages et puits) dans trois cercles de la région de Koulikoro, Mali
}

\section{The bacteriological quality of the wters of consumption (drillings and Well) of three circle of the region of Koulikoro, Mali}

\author{
Coumare $\mathrm{K}^{1}$, Diallo $\mathrm{T}^{1,2}$, Siby $\mathrm{L}^{1}$, Haidara $\mathrm{A}^{1}$, Traore $\mathrm{M}^{1}$, Coulibaly $\mathrm{M}^{1}$,Sangare $\mathrm{D}^{1}$, Traore $\mathrm{IT}^{1}$, Tangara $\mathrm{D}^{1}$, \\ Coulibaly $\mathrm{SM}^{1}$, Koumare BY $\mathrm{Y}^{1,2}$ \\ ${ }^{1}$ Ministère de la Santé et de l'Hygiène Publique, Laboratoire National de la Santé. \\ ${ }^{2}$ Faculté de Pharmacie, Université des Sciences, des Techniques et des Technologies de Bamako, Mali. \\ $\underline{\text { Auteur correspondant }}$ : Docteur Tidiane Diallo, Maître Assistant en Toxicologie, Département Sciences du \\ Médicament, Faculté de Pharmacie, Université des Sciences, des Techniques et des Technologies de Bamako, \\ Mali. Email : tidiallo2017@gmail.com
}

\section{RESUME}

Objectifs : Contrôler la qualité bactériologique des eaux souterraines à usage alimentaire « consommation » de la région de Koulikoro en vue d'améliorer l'état de santé de la population.

Matériel et Méthodes : Notre méthode était basée sur la recherche qualitative et quantitative des coliformes thermo tolérant et des Escherichia Coli. Nous avons utilisés les milieux de culture suivants : Violet Rouge Bililé Lactosé (VRBL) pour isoler les coliformes et Tryptone Bile X-Glucuronide Agar (TBX) pour isoler les Escherichia Coli. Les colonies ont été dénombrées après incubation. La conformité des échantillons a été mise en évidence travers les normes françaises V08-060 pour les Coliformes Thermo tolérant et ISO 16649-2 pour les Escherichia coli.

Résultats : Durant notre étude nous avons collecté 172 échantillons de $900 \mathrm{ml}$ d'eau à travers 80 puits et 92 forages répartis comme suit : 20 à Banamba (Touba Sylla), 92 à Dioila (N'Golobougou, N'Tobougou, Siankoro, Dégnekoro) et 60 à Kati (Kambila, Koursalé, Tinkelé). Sur les 172 échantillons, 75 étaient non conforme soit un taux de $43,6 \%$.

Les eaux de puits étaient plus contaminées avec $94,7 \%$ de non-conformité contre $5,3 \%$ pour les eaux de forage. Le cercle de Dioila a enregistré plus de cas de non-conformité $(53,3 \%)$. Les deux microorganismes ont été tous les 2 sources deI. contaminations des eaux non conformes.

Conclusion : L'éducation et la sensibilisation de la population sur les bonnes pratiques d'hygiène publique autours des eaux doivent être mise en évidence afin d'éviter les maladies hydriques.

Mots clés : eau, qualité, contaminant biologique, Koulikoro.

\section{Summary}

Summary

Objective: Bacteriological Control quality of groundwater intended for the consumption in Koulikoro region in Mali in order to improve the health of the local population.
Materiel and Methods: For our analyzes, we used the following materials: $1 \mathrm{~mL}$ pipette, petri dish, thermostatic bath, thermometer, $\mathrm{pH}$ meter and electronic balance. Our method was based on the qualitative and quantitative research of thermotolerant coliforms and Escherichia coli. The culture media used were as follows: "Violet Rouge Bililé Lactose (VRBL)" to isolate coliforms and "Tryptone Bile X-Glucuronide Agar (TBX)" to isolate Escherichia Coli. After incubation, the colonies were counted to determine the conformity of the samples where we used French standards NF V08-060 for Coliforms Thermo-tolerant and NF EN ISO 16649-2 for Escherichia coli.

Results : During our study, we collected 172 samples by collecting $900 \mathrm{ml}$ of water samples in 80 wells and 92 drillings in the Koulikoro area. Among our 172 samples, 75 or $43.6 \%$ were not in conformity and could be due to the non-protection of the wells and the absence of the basic hygiene rules. Well water was more contaminated with $94.7 \%$ of non-conformity than drilling waters of $5.3 \%$. Dioila area recorded more cases of nonconformity $(53.3 \%)$ followed by Kati (26.7) and Banamba (20\%). Escherichia Coli and Coliforms Thermo-tolerant have been both sources of contamination of non-conformity waters.

Keywords: Water, Quality, Biological contamination, Koulikoro.

INTRODUCTION

L'eau est la ressource naturelle grâce à laquelle se développe et se maintient la vie. Malgré son abondance, couvrant $70 \%$ de la surface de la terre, l'accès à l'eau potable constitue un problème majeur de santé publique [1]. Au Mali, comme dans la plupart des pays en développement la gestion des ressources en eaux devient de plus en plus problématique.

En 2010, 783 millions de personnes n'avaient pas accès à l'eau potable provenant de sources améliorées [2].

Les eaux de mauvaise qualité peuvent véhiculer plusieurs microorganismes et substances toxiques organiques et inorganiques appelés «polluants de l'eau ». 
Généralement sources de maladies dites hydriques, ces polluants surtout microbiologiques (Escherichia Coli, Salmonelles, Légionnelles...) sont responsables d'épidémies qui menacent la santé des populations. Dans nos pays ces microorganismes ne sont pas que bactériens, peuvent aussi être virales (Poliomyélitique, Coxsackie $\mathrm{A}, \ldots$ ) mais très souvent parasitaires (nématodes, cestodes,(i) trématodes,...).

À l'échelle mondiale, la pollution bactériologique (choléra, typhoïdes, diarrhées d'origine autres(ii) microorganismes...) des eaux est l'une des principales causes de décès. Selon, le rapport de Nations Unies (2012), les maladies infectieuses engendrées par les microorganismes comme la pneumonie et la diarrhée étaient de loin l'une des principales causes de mortalité à l'échelle mondiale [3]. En Afrique subsaharienne plus de $40 \%$ des personnes n'ont pas accès à eau potable améliorée [3]. De plus, vers la fin de l'année 2000, la diarrhée à elle seule a tué environ cinq millions de personnes à travers le monde dont 3,3 millions d'enfants de moins de cinq ans [4]. Au Mali, selon l'Enquête Démographique et de Santé (EDSM-V) 2012-2013, les maladies diarrhéiques liées à l'eau de consommation étaient de $16,4 \%$ chez les enfants de moins de 5 ans [5].

Au Mali en 2004, trois millions de la population rurale n'avaient pas accès suffisant à l'eau potable. $\mathrm{Au}$ total, plus de 10000 points d'eau modernes étaient encore à installer dans les campagnes maliennes pour que tous les besoins en eau domestique soient satisfaits [6]. Il a été estimé au Mali que $38 \%$ des ménages utilisent l'eau provenant des puits publics à ciel ouvert. Cette proportion est de $14 \%$ pour les puits individuels, non protégés. L'insuffisance des ouvrages, d'assainissement et la méconnaissance des règles élémentaires d'hygiène favorisent la propagation des maladies féco-orales [6].

Toutefois, l'atteinte de l'objectif numéro VI, du développement durable 2016-2030 (OMS, entité eau potable et assainissement) : "garantir l'accès de tous à l'eau et à l'assainissement et assurer une gestion des ressources en eau», nécessite des évaluations continues de la qualité de l'eau de consommation au Mali. L'objectif de cette étude est d'évaluer les conditions d'exploitations et la qualité microbiologique des eaux souterraines (forages et puits) destinée à l'usage domestiques dans trois cercles de la région de Koulikoro.

\section{MATÉRIELS ET MÉTHODE}

\section{Type et période d'étude}

Il s'agit d'une étude prospective à visée analytique. Elle a été réalisée durant le mois de mars 2017 dans trois cercles de la région de Koulikoro : Banamba (Touba Sylla), Dioila (N'Golobougou, N'Tobougou, Siankoro, Dégnekoro) et Kati (Kambila, Koursalé, Tinkelé), Mali.

\section{Echantillonnage}

L'échantillonnage a été réalisé en fonction des sources en eau de forages et de puits disponibles dans les huit communes rurales des trois cercles de la région de Koulikoro La collecte des échantillons a été effectuée conformément au processus préétablie :

administration du questionnaire au chef de famille ou à son représentant(e) et au gestionnaire des forages et puits dans les différents sites d'étude ; prélèvements des eaux de forages et de puits.

Les questions administrées étaient relatives aux mesures de protections des forages et puits, aux moyens de transport et de conservation des eaux. Les eaux de forages ont été directement prélevées à la borne fontaine à l'aide des flacons stériles de 900 $\mathrm{mL}$ tandis que les eaux de puits ont été prélevées par puisage à l'aide de puisards de laboratoire (flacons stériles fixés à une corde). Nous avons prélevés $900 \mathrm{~mL}$ d'eau de forage et de puits. Après le prélèvement chaque échantillon a été codifié et sceller pour analyse microbiologique au laboratoire. Analyse microbiologique des eaux

Pour les analyses, nous avons utilisé les matériaux suivants : pipette de $1 \mathrm{~mL}$ stérile, boites de pétri, bain marie thermostatique, thermomètre, $\mathrm{pH}$-mètre, Balance électroniques.

Nos analyses ont été réalisées au service de bactériologie du Laboratoire National de la Santé, une structure publique à caractère scientifique et technologique dotée d'une accréditation en microbiologique conformément aux normes internationales ISO/CEI 17025 [8].

La technique analytique était basée sur la recherche qualitative et quantitative des coliformes thermo tolérant et des Escherichia coli dans nos échantillons d'eaux prélevées.

\section{Préparation des milieux de culture}

Deux milieux de cultures étaient utilisés e Violet Rouge Billé Lactosé (VRBL) pour isoler les coliformes thermo tolérant et Tryptone Bile XGlucuronide Agar (TBX) pour isoler les Escherichia Coli (voir figure 1).

Pour le premier milieu de culture, 35,5g de VRBL étaient dissous dans $1 \mathrm{~L}$ d'eau distillée, puis porté le mélange à l'ébullition sans dépasser 2 minutes, tout en agitant fréquemment jusqu'à la dissolution complète du VRBL. Cependant, le mélange ne doit pas être porté à l'autoclave, ni surchauffer car ces processus peuvent entrainer la dégradation de la bile. La solution préparée est mise au bain de surfusion pendant 4 heures à une température comprise entre $46^{\circ} \mathrm{C}$. Le $\mathrm{pH}$ final obtenu après ajustement avec du soude caustique était de 7,4 à $25^{\circ} \mathrm{C}$

Le deuxième milieu de culture a été préparé en dissolvant $36.6 \mathrm{~g}$ de TBX dans $1 \mathrm{~L}$ d'eau distillée puis porté le mélange à l'ébullition tout en agitant vigoureusement jusqu'à dissolution complète. La solution obtenue a été porté à l'autoclave à $121^{\circ} \mathrm{C}$ 
pendant 15 min. Le $\mathrm{pH}$ final était de 7,2 à $25^{\circ} \mathrm{C}$ dans ce milieu.

\section{Ensemencement}

Nous avons pipeté $1 \mathrm{ml}$ de notre échantillon ou inoculum, puis déposé ce volume dans deux boites de pétri conformément aux procédés de la norme 17025 [7]. Nous avons ainsi coulé 15 à $20 \mathrm{ml} \mathrm{du}$ milieu de culture en deux étapes une première couche, après une deuxième couche et laisser solidifier le milieu de culture après avoir homogénéise.

Les boites de pétri ont été incubé à $44^{\circ} \mathrm{C}$ pendant 24h (température adéquat pour la multiplication des coliformes thermo tolérant conformément à la norme française V08-060), [9] et NF EN ISO 16649-2 pour les Escherichia coli [10] voir figure 2.

Lecture et méthode de calcul des colonies

Nous avons dénombré les colonies à l'aide d'un conteur électronique de colonies. Les colonies des coliformes thermo tolérant sur le milieu VRBL sont de couleur rouge-violé foncé tandis que les colonies des Escherichia colis sont de couleur bleue dans le milieu TBX.

Le résultat a été exprimé selon les normes françaises 7218 et $9308-1$ pour les deux germes pathogènes $[11,12]$.

Le nombre des colonies a été déterminé selon la formule ci-dessous [12]:

prélevés dans les différentes localités est montrée dans la figure 3 .

Condition d'exploitation des eaux de puits et forages

Sur les sites d'étude l'ensemble des puits étaient de réalisation traditionnelle avec une profondeur comprise entre 5 à 15 mètres. La majorité soit $64 \%$ ne disposait pas de systèmes de protection. Le reste était muni de simple protection constituée de pneus d'une hauteur d'environ 20 à $30 \mathrm{~cm}$ avec des couvercles faites de tôle et/ou bois.

Les forages étaient convenablement protégés avec des bornes munies de pompes manuelles.

Après prélèvement des eaux dans les deux sources, la population transportait et stocker les eaux dans des seaux $(61 \%)$, des bidons $(59 \%)$ et des bassines (52\%). Ces eaux servaient à des usages domestiques (préparation des aliments, vaisselles,...) et à la consommation (abreuvage humaine et animale).

Contrôle qualité microbiologique des eaux de puits et forages

Les analyses microbiologiques étaient non conformes dans $43,6 \%$ (75/172) des cas.

Les eaux de puits étaient plus contaminées avec $94,7 \%$ de non-conformité contre $5,3 \%$ pour les eaux de forage. Les eaux de forage contaminées provenaient uniquement du cercle de Dioila: communes de N'Golobougou et Siankoro.

Le cercle de Dioila a enregistré plus de cas de non-

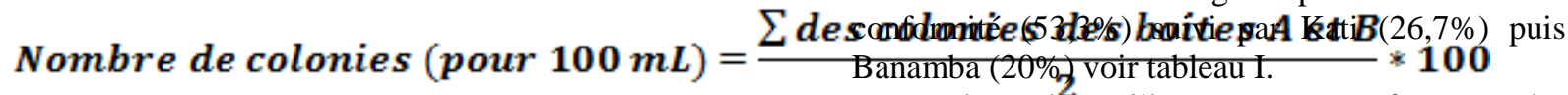

- $\quad \sum$ des colonies (des boites A et B)/2: C'est la moyenne du nombre des colonies comptés dans la boite de pétri A et la boite de pétri B

- Le résultat est exprimé en pourcentage d'où la multiplication par 100

Le résultat est exprimé en Unité de Format Colonie (UFC) pour $100 \mathrm{~mL}$ d'échantillon.

Les échantillons exempt de colonies $(\mathbf{0} / \mathbf{1 0 0} \mathbf{m L})$ ont été considéré conformes et ceux montrant une présence quantitative et qualitative de Coliformes thermo tolérant ou Escherichia colis étaient IV. considérés comme non conforme.

Analyses statistiques

Le logiciel Epi-info version 7 a été utilisé pour l'analyse les données. Le masque de saisie a été élaboré dès la validation du questionnaire.

Considération éthique et analyse statistique

Nous avons obtenu l'avis favorable des chefs de familles et les responsables chargés de la gestion des puits et/ou forage pour la réalisation de l'étude. L'anonymat et la confidentialité ont été garantis pour tous les échantillons à travers leur codification.

\section{RÉSULTATS}

Nous avons collecté 172 échantillons provenant des forages et puits respectivement $53 \%$ et $47 \%$ durant cette étude. . La répartition des échantillons
Dans les échantillons non conformes, les Coliformes thermo tolérant étaient majoritairement observés avec 73/75 des échantillons contre 21/75 des échantillons pour Escherichia coli_avec un taux de co-contamination de $28 \%$.

L'unité de format de colonie par échantillon était variable en fonction de la source et de la localité, elle variait de 100 à 3650 pour Escherichia coli et de 100 à plus de 15000 pour les Coliformes thermo tolérant voir tableaux II et III.

\section{DISCUSSIONS}

Cette étude a été réalisée dans huit zones rurales (Touba Sylla, N'Golobougou, N'Tobougou, Siankoro, Dégnekoro, Kambila, Koursalé, Tinkelé) de Koulikoro, deuxième région administrative du Mali. Le choix de ces lieux d'étude se justifie par la forte potentialité des points d'eau traditionnels du Mali tels que les puits dans la région de Koulikoro (36\%) [13]. De plus, l'étude visait une évaluation de la qualité des eaux de forage et puits existant en vue d'une implantation des forages dans les zones où les eaux seront impropres à la consommation dans la région de Koulikoro.

L'analyse microbiologique a été effectuée sur 172 échantillons d'eau provenant de 92 forages et 80 puits prélevés sur les différents sites d'étude. Ce nombre représente l'effectivité des forages et puits 
qui sont des sources d'exploitation en eau pour des usages domestiques dans les sites d'étude.

$\mathrm{Au}$ Mali, les résultats d'une étude sur le plan national d'accès à l'eau potable révèlent que $83 \%$ des eaux de puits utilisées provenaient des puits traditionnels [12]. Notre travail confirme ces résultats car la totalité de nos puits évalués étaientV. de construction traditionnelle. Par ailleurs, les eaux de forages ont constitué $53 \%$ de nos échantillons. Cela s'explique par la réalisation de plus en plus récente des forages par rapport aux puits. Ainsi, depuis quelques années la réalisation de forages tend à se développer malgré son coût élevé. Ils servent essentiellement à l'alimentation en eau potable et à d'autres usages domestiques. Ils équipent aussi de nombreuses infrastructures d'éducation et sanitaires.

Avant le prélèvement et l'analyse des échantillons nous avons fait des essais de vérification d'absence totale des deux micro-organismes (Coliformes thermo tolérant et Escherichia coli) dans les matériaux utilisés par l'utilisation d'équipement et matériel stérilisés et à usage unique. Le principe de vérification est basé sur le contrôle de stérilité et d'inhibiteur du matériel d'analyse par ensemencement en profondeur du milieu de culture puis on incube après on fait la lecture. S'il n'existe pas de germes nous concluons que le matériel est stérile. Le choix de ces deux microorganismesVI. s'explique par le fort taux de mortalité et de morbidité lié aux maladies diarrhéiques d'origine microbienne à l'échelle mondiale [3,4].

La forte contamination des eaux de puits $(94,7 \%)$ observée dans ce travail pourrait être due à la non protection des puits et le manque de mesures d'hygiène lors du transport et le stockage des eaux. En plus il y avait un manque de protection des eaux lors du transport dans les seaux et basine. Les différences de non-conformité dans les localités étaient liées à la répartition disportionnée (forages et puits) ainsi que l'hygiène environnementale dans ces différents sites d'étude.Ces facteurs de contamination ont été apportés dans de nombreuses études [14-17].

Les systèmes de protection des puits étaient constitués par de pneus d'une hauteur 20 à 30 centimètres et des couvercles en tôle ou en bois. Tandis que les forages étaient munis d'une borne fontaine exploitable à l'aide d'une pompe manuelle et fermée, ce qui lui confère une protection optimale. Sur les huit communes seules les eaux de la commune de Kambila étaient conformes.

Les Coliformes thermo tolérant et Escherichia coli ont tous deux été retrouvé comme sources de contaminations avec un fort taux pour les Coliformes thermo tolérant avec $97 \%$ de contamination des échantillons non conforme. Dans certains échantillons les deux micro-organismes étaient présents à des proportions UFC différentes. Nos résultats étaient différents de ceux
d'Heriarivony S. et al qui ont trouvé une UFC de Coliformes thermo tolérant inférieur à un [18]. Cependant, au Bénin, Cyriaque D. et al. ont trouvé une contamination à $100 \%$ des échantillons pour les deux micro-organismes dans des eaux de puits, confortant nos données [19].

\section{CONCLUSION ET PERSPECTIVES}

Notre étude a révélé un taux de non-conformité élevé pour la qualité bactériologique (Coliformes thermo tolérant et Escherichia coli) des eaux analysées en particulier pour les eaux de puits. Ces eaux hors normes constituent un risque pour la santé des populations. Les résultats obtenus invitent à un suivi régulier de la qualité des différents types d'eau et la prise de dispositions spécifiques pour éviter la pollution des eaux à usage domestique. Pour les eaux de puits, des dispositions règlementaires doivent être prises en pour le choix judicieux de leurs emplacements et de leurs protections contre les pollutions diverses.

La qualité acceptable des eaux de forage doit être renforcée en améliorant leurs modes de réalisation et une gestion adéquate des eaux usées.

Des dispositions pratiques par l'éducation et la sensibilisation de la population par rapport aux bonnes pratiques d'hygiène autour de ces eaux doivent être mise en évidence afin d'éviter les maladies hydriques.

\section{RÉFÉRENCES BIBLIOGRAPHIQUES}

1. LENNTECH B.V. FAQ sur la quantité d'eau: quelle qualité d'eau y a-t-il sur terre ? Consulté le 21 juillet 2017 sur l'URL : http://www.lenntech.fr/faq-eau-quantite-1.htm

2. WATERAID, 2013. Partout et pour tous. Une vision pour l'accès à l'eau potable, à l'hygiène et à l'assainissement après 2015. WaterAid, Londres, Royaume-Uni

3. NATIONS UNIES. Assurer un environnement durable. Objectifs du Millénaire pour le développement, rapport 2012. New York 12-24533Juin 2012-2000

4. HOEK, W., KONRADSEN, F. \& JEHANGIR, W. A. 1999. Domestic Use of Irrigation Water: Health Hazard or Opportunity? Water Resources Development, 15:1-2

5. Cellule de Planification et de Statistique (CPS/SSDSPF), Institut National de la Statistique (INSTAT/MPATP), INFO-STAT et ICF International, 2014. Enquête Démographique et de Santé au Mali 2012-2013. Rockville, Maryland, USA : CPS, INSTAT, INFO-STAT et ICF International.

6. Association Forage Mali. Guide pour la Réalisation de Forages au Mali. Consulté le 14 septembre 2017 sur

l'URL : http://www.pseau.org/outils/ouvrages/forages_mali _guide_pour_la_realisation_de_forages_au_mali_2 011.pdf

7. Norme européenne et française. Organisation Internationale de Standardisation et Commission 
Electronique Internationale 17025. Exigences générales concernant la compétence des laboratoires d'étalonnages et d'essais. Edité et diffusé par l'Association Française de Normalisation (AFNOR) 29 nov. 2005. Deuxième tirage 2005-10-P

8. Organisation Internationale de Standardisation 11133. Microbiologie des aliments, des aliments pour animaux et de l'eau : Préparation, production, stockage et essais de performance des milieux de culture. Première édition 15 mai 2014, version corrigée 01 nov. 2017 en Suisse.

9. Norme Française V08-060. Microbiologie des aliments: Dénombrement des coliformes thermo tolérants par comptage des colonies obtenues à $44^{\circ} \mathrm{C}$. Edition Avril 2009. Chapitre mode opératoire 9.2.2 Page 8.

10. Organisation Internationale de Standardisation 16649-2. Microbiologie des aliments: méthode horizontale pour le dénombrement des Escherichia coli $\beta$-glucuronidase positive. Partie 2 : Technique de comptage des colonies à $44^{\circ} \mathrm{C}$ au moyen de 5 bromo-4-chloro-3-indolyl $\beta$-D-glucuronate. Première édition 05 avr. 2001. Imprimé en Suisse.

11. Organisation Internationale de Standardisation 7218. Microbiologie des aliments: Exigences générales et recommandations. Troisième édition 15 aout 2007, Amendement 1 du 01 aout 2013. Publié en Suisse.

12. Organisation Internationale de Standardisation 9308-1 Septembre 2000.Qualité de l'eau Recherche et dénombrement des Escherichia coli et des bactéries coliformes.

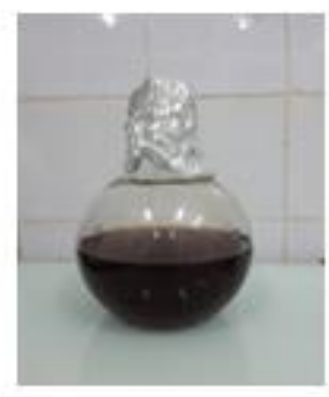

13. Ministère des Mines de L'Energie et de l'Eau. Plan national d'accès à l'eau potable 2004-2015. Consulté le 16 septembre 2017, consultable sur l'URL :

http://www.pseau.org/sites/default/files/fichiers/mal $\mathrm{i} / 4-$

_Plan_national_d_acces_a_1_eau_potable_20042015.pdf

14. Cazaban M, Duffour J, Fabbro-Peray P. Santé publique. $5^{\text {ème }}$ édition. Paris : Masson, 2005.

15. Arnaud M, Hartemann P. Qualité de l'eau et santé. Lettre scientifique IFN 1996 ; $43: 5-10$.

16. Centre d'expertise en analyse environnementale du Québec. Recherche et dénombrement des bactéries hétérotrophes aérobies et anaérobies facultatives ; méthode par incorporation à la gélose. Québec : Gouvernement du Québec, 2004.

17. Groupe scientifique sur l'eau. Coliformes fécaux. Fiches synthèses sur l'eau potable et la santé humaine. Québec : Institut national de santé publique du Québec, 2003.

18. Heriarivony S. C., Razanamparany B., Rakotomalala J. E. Caractères Physico-Chimiques et Bactériologiques de l'eau de consommation (Puits) de la Commune Rurale d'Antanifotsy, Region Vakinankaratra, Madagascar. Larhyss Journal, ISSN 1112-3680, $n^{\circ} 24$, Décembre 2015, pp. 7-17.

19. Cyriaque Dégbey, Michel Makoutode, EdgardMarius Ouendo, Benjamin Fayomi, Christophe De Brouwer. La qualité de l'eau de puits dans la commune d'Abomey-Calavi au Bénin. Environnement, Risques \& Santé - Vol. 7, $\mathrm{n}^{\circ} 4$, juillet-août 2008 .

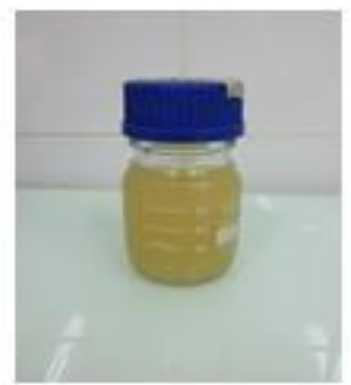

Figure 1 : Milieux de culture avant incubation VRBL (à gauche) et TBX (à droite).
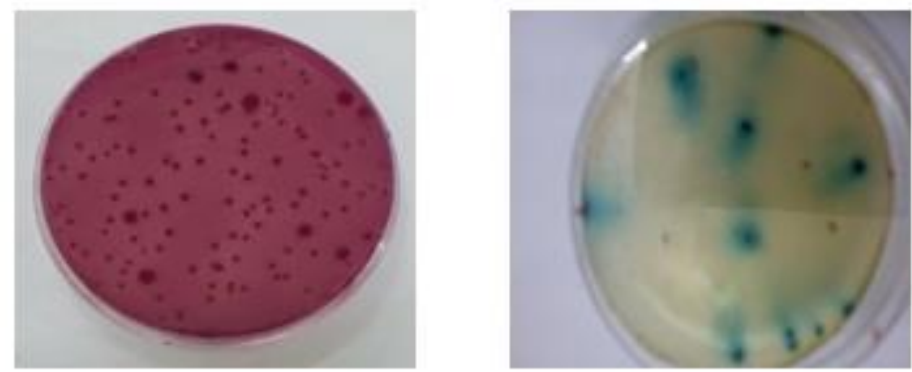
Figure 2 : Milieux de cultures après incubation, VRBL contenant les colonies Coliformes thermo tolérant (à gauche) et TBX contenant les colonies d'Escherichia coli (à droite).

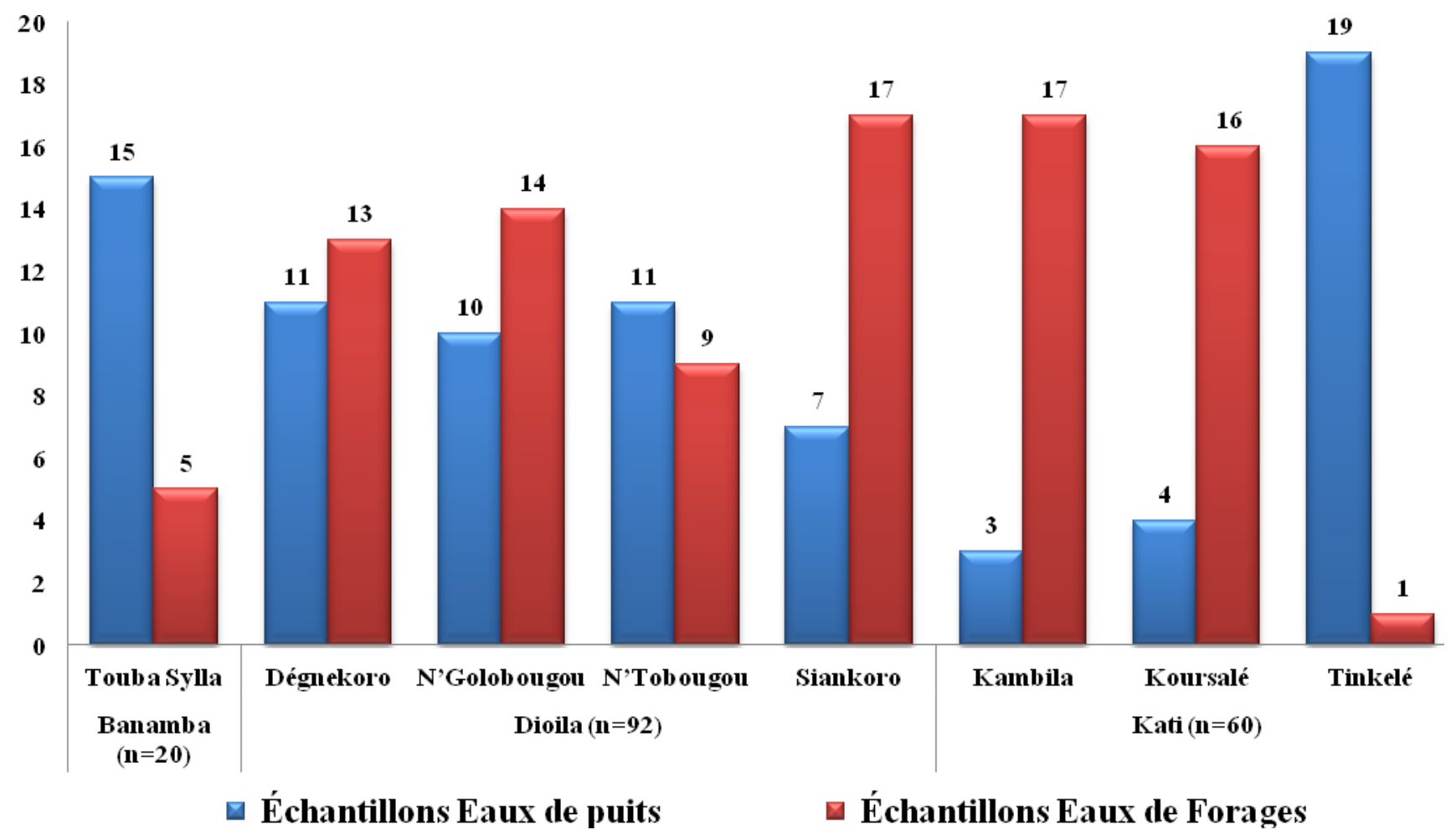

Figure 3 : la répartition des échantillons selon les sources d'eaux et par localités

Tableau I : Taux de non conformes selon les localités et la nature des eaux

Nombre d'échantillons non conforme

Cercles Communes Eaux de puits (\%) Eaux de Forages (\%)

Banamba (n=15/20) Touba Sylla 15(100) 0

Dégnekoro 11(100) 0

$\begin{array}{llll}\text { Dioila (n=40/92) } & \text { N'Golobougou } & 09(90) & 1(7) \\ & \text { N'Tobougou } & 11(100) & 0\end{array}$




\begin{tabular}{llll}
\hline & Siankoro & $05(71)$ & $3(18)$ \\
\hline & Kambila & 0 & 0 \\
Kati (n=20/60) & Koursalé & $02(50)$ & 0 \\
& Tinkelé & $18(95)$ & 0 \\
\hline
\end{tabular}

Tableau II : Répartition des Unité de Format de Colonie d'Escherichia coli pour 100mL d'échantillon en fonction des localités.

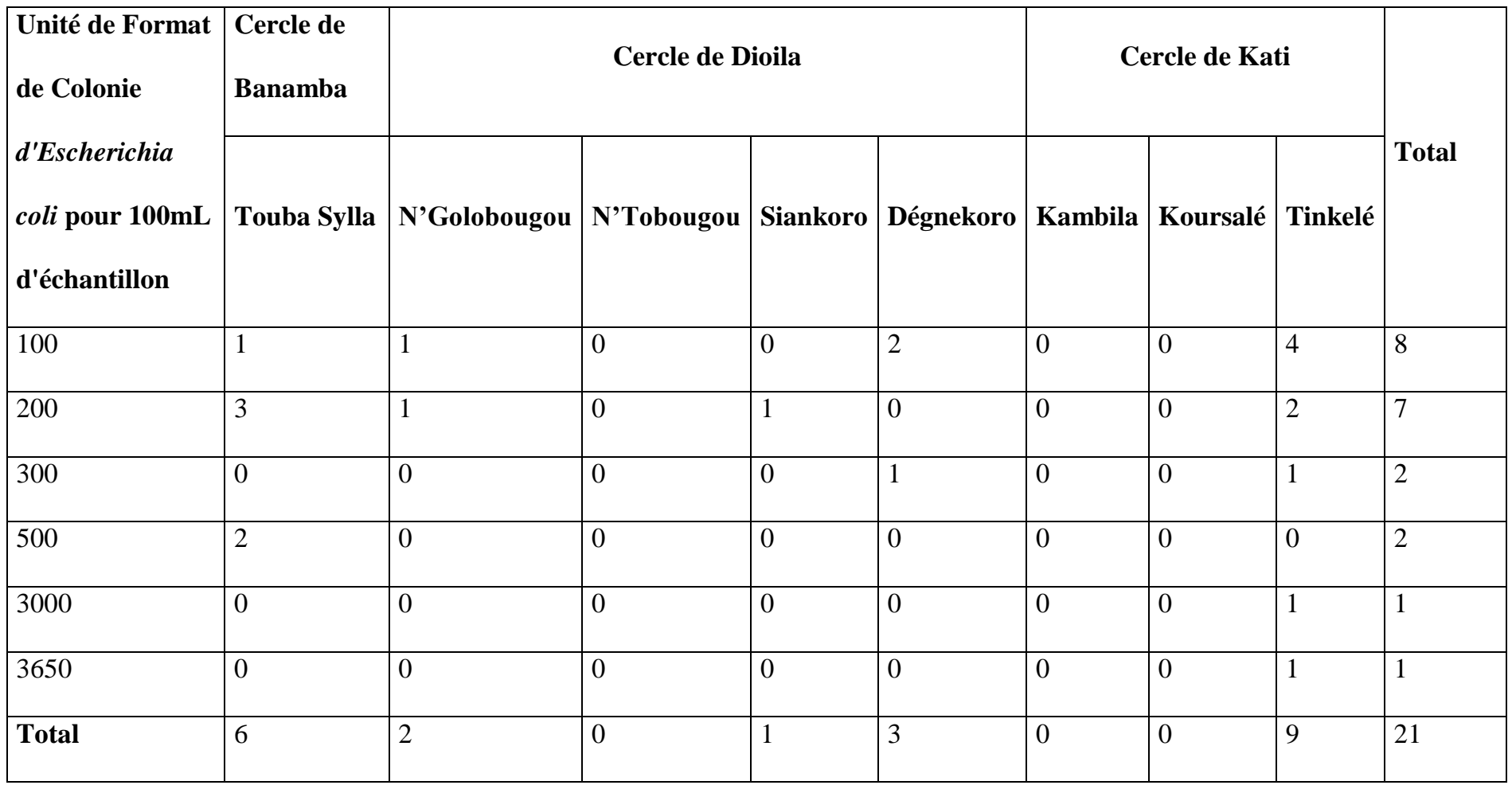


Tableau III : Répartition des Unité de Format de Colonie des Coliformes Thermo tolérants pour $100 \mathrm{~mL}$ d'échantillon en fonction des localités

\begin{tabular}{|c|c|c|c|c|c|c|c|c|c|}
\hline \multirow{2}{*}{$\begin{array}{l}\text { Unité de Format } \\
\text { de Colonie de } \\
\text { Coliforme } \\
\text { Thermo tolérant } \\
\text { pour } 100 \mathrm{~mL} \\
\text { d'échantillon }\end{array}$} & $\begin{array}{l}\text { Cercle de } \\
\text { Banamba }\end{array}$ & \multicolumn{4}{|c|}{ Cercle de Dioila } & \multicolumn{3}{|c|}{ Cercle de Kati } & \multirow[b]{2}{*}{ Total } \\
\hline & Touba Sylla & N'Golobougou & N'Tobougou & Siankoro & Dégnekoro & Kambila & Koursalé & Tinkelé & \\
\hline 100 & 5 & 2 & 4 & 5 & 2 & 0 & 1 & 3 & 22 \\
\hline 200 & 2 & 1 & 3 & 2 & 3 & 0 & 1 & 1 & 13 \\
\hline 300 & 2 & 3 & 1 & 0 & 0 & 0 & 0 & 2 & 8 \\
\hline 400 & 1 & 0 & 0 & 0 & 0 & 0 & 0 & 1 & 2 \\
\hline 500 & 0 & 1 & 1 & 0 & 0 & 0 & 0 & 1 & 3 \\
\hline 550 & 0 & 0 & 0 & 0 & 0 & 0 & 0 & 1 & 1 \\
\hline 600 & 0 & 2 & 1 & 0 & 0 & 0 & 0 & 0 & 3 \\
\hline 700 & 1 & 0 & 0 & \begin{tabular}{|l|l|}
0 \\
\end{tabular} & 0 & 0 & 0 & 0 & 1 \\
\hline 800 & 0 & 0 & 0 & 0 & 0 & 0 & 0 & 1 & 1 \\
\hline 1000 & 1 & 0 & 0 & 0 & 0 & 0 & 0 & 0 & 1 \\
\hline 1100 & 0 & 0 & 0 & 0 & 0 & 0 & 0 & 1 & 1 \\
\hline 1200 & 0 & 0 & 1 & 0 & 0 & 0 & 0 & 0 & 1 \\
\hline 2650 & 0 & 0 & 0 & 0 & 0 & 0 & 0 & 1 & 1 \\
\hline 2800 & 0 & 0 & 0 & 0 & 1 & 0 & 0 & 0 & 1 \\
\hline 3650 & 0 & 0 & 0 & 1 & 0 & 0 & 0 & 0 & 1 \\
\hline 5450 & 0 & 1 & 0 & 0 & 0 & 0 & 0 & 0 & 1 \\
\hline 6450 & 0 & 0 & 0 & 0 & 0 & 0 & 0 & 1 & 1 \\
\hline 8200 & 0 & 0 & 0 & 0 & 1 & 0 & 0 & 0 & 1 \\
\hline 9000 & 0 & 0 & 0 & 0 & 1 & 0 & 0 & 0 & 1 \\
\hline 9400 & 0 & 0 & 0 & 0 & 1 & 0 & 0 & 0 & 1 \\
\hline$\geq 15000$ & 3 & 0 & 0 & 0 & 1 & 0 & 0 & 4 & 8 \\
\hline Total & 15 & \begin{tabular}{|l|}
10 \\
\end{tabular} & 11 & 8 & 10 & 0 & 2 & \begin{tabular}{|l|}
17 \\
\end{tabular} & 73 \\
\hline
\end{tabular}

